\title{
Liquid Jet Decelerates Faster than Expected
}

\author{
Video recordings show that the small mountain of liquid that appears \\ after a drop hits a liquid surface has some surprising properties. \\ By Griffin Wilce
}

V

ideo of a "jet"-the small spike that forms just after a drop hits a larger body of liquid-shows that the top of the structure initially decelerates at up to 20 times the gravitational acceleration $g$ [1]. This rapid deceleration suggests that surface tension, not gravity, is the driving force behind the shapes of jets.

Cees van Rijn of the University of Amsterdam and his colleagues released single drops of liquid containing fluorescent particles from heights of $8-33 \mathrm{~cm}$ above a bath of liquid that contained the same particles. The researchers tested water, ethanol, and a glycerol-water mixture. Using laser illumination, they recorded the motion of the particles to find the velocity and shape of the

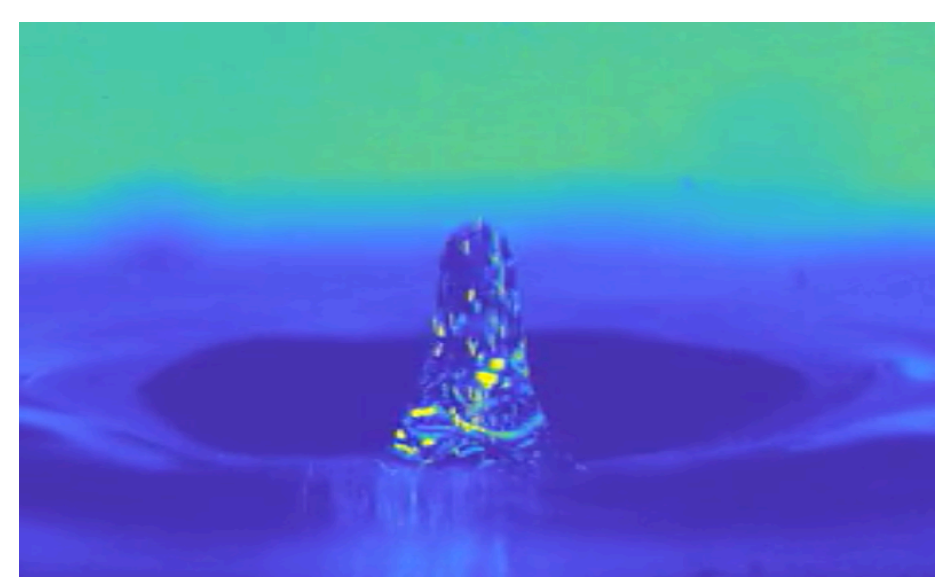

Pulling $g$ 's in a jet. The tip of this liquid jet, created by a drop of liquid hitting the surface moments earlier, initially decelerates at 20 times the gravitational acceleration $g$.

Credit: C. J. M. van Rijn et al. [1]

jet.

The most extreme deceleration occurred in the jet's earliest moments, but the deceleration dropped to $g$ by the time the jet fell back to the surface. The researchers also found that the jet shape was self-similar, meaning that the shape remained essentially unchanged during most of its existence, other than a time-dependent scaling factor for the horizontal and vertical

A false-color video of the formation of a jet in water, with fluorescent particles dispersed throughout. Time is in milliseconds. Video is slowed down by 10 times.

Credit: C. J. M. van Rijn et al. [1] axes. The researchers developed a theoretical model that agreed with the data for a wide range of fluid viscosities, densities, and surface tensions. For their next steps, van Rijn and his colleagues would like to perform similar experiments on the International Space Station or in another low-gravity environment in order to observe the effects of surface tension 
on the formation of the jet, independent of gravity.

Griffin Wilce is a Physics science writing intern.

\section{REFERENCES}

1. C. J. M. van Rijn et al., "Self-similar jet evolution after drop impact on a liquid surface," Phys. Rev. Fluids 6, 034801 (2021). 